\title{
A Management Model for Integrating SOA and XBRL
}

José Rogério Poggio Moreira. Universidade Salvador - UNIFACS. Brasil rogerio.poggio@gmail.com

Paulo Caetano da Silva. Universidade Salvador - UNIFACS. Brasil paulo.caetano@ pro.unifacs.br

\begin{abstract}
The alignment and adaptation of business processes with organizational strategies require a high level of effort affecting several businesses. This is caused by the low level of flexibility in information systems to changes in business requirements. Another problem encountered in organizations is the diversity of data formats that are related to financial information, since these are stored in heterogeneous computer systems. An adaptive and structured IT environment can influence the improvment of flexibility and adaptability of business processes, including information systems. Based on this context and with a view to solving these problems, we propose in this paper a model of SOA (service-oriented architecture) deployment and integration with XBRL. This work is expected to contribute to the ease of adaptation of information systems to business changes and improvements in the quality and reliability of financial reporting issues.
\end{abstract}

Keywords: SOA Governance, SOA, XBRL.

\section{INTRODUCTION}

Currently, format diversity in financial report data is affecting diverse organizations. This is occurring because data are placed in different information systems, which causes extraction difficulty and the need for data transformation and processing to promote information consolidation in financial reports. This increases significantly: (i) the emission time of financial reports, due to data transformation efforts; (ii) the cost originating from these performance efforts; and (iii) error risk, thus decreasing the reports' reliability and generating, as a consequence, negative impacts for the business. These problems related to format 
diversity in financial information data may result in incomplete financial reports, as well as reports without the necessary precision to provide the transparency required by laws and regulations, and do not allow the performance of accurate financial analysis, therefore causing insecurity in the market and a lack of adherence to legal requirements.

In an effort to solve these issues, the Extensible Business Reporting Language - XBRL (XBRL, 2003) was developed, which is an international standard adopted by public and governmental organizations that contributes to integrating financial information distributed in different data formats (Silva, 2003).

The service-oriented architecture (SOA) provides information systems and business processes with better adaptation to the changes that take place in business requirements (Erl, 2009). In this work context, the SOA has the goal of creating services that will automatize the business processes related to the organization's financial and accounting areas, enabling applications to be more flexible to the changes and aligned with the business processes that promote financial reports' emission.

$\mathrm{XBRL}$ is an international facto pattern, initially developed for the accounting and financial areas and focusing on reports' emission (Cruz et al., 2012). However, nowadays XBRL is also involved in non-financial initiatives, such as Global Reporting Initiatives (GRI, 2013), statistical reporting and so on. Another factor contributing to its adoption is the fact that its usage results in the following benefits (HM Revenues \& Customs - UK Government, 2009): (i) smaller error possibility, due to minimizing the need for data re-entry; (ii) better quality of the data received; (iii) the facility of data manipulation in information systems; (iv) financial report reliability and security increase; and (v) an improvement in data transference and communication among different companies' departments and offices. SOA has been adopted worldwide due to its capacity to adapt constructed services easily to the changes that occur in organizations' business processes, besides the greater service alignment provided by the information technology area with the business processes, allowing these processes to evolve without generating negative impacts for organizations.

The rest of this paper is organized as follows. Section 2 discusses the concepts related to the SOA. Section 3 analyses the definitions and concepts of XBRL technology. Section 4 details the structure of the SOA implantation model and 
integration with XBRL. In Section 5, some usage scenarios of the proposed model are presented. Finally, the conclusion and further considerations related to this paper are presented.

\section{SOA (SERVICE-ORIENTED ARCHITECTURE)}

SOA is a software architecture that allows the availability of functionalities implemented by applications like services, which are software based on distributed systems architecture that accepts requisitions and gives back responses. SOA enables a connection among created services through a services bus or enterprise service bus (ESB), aiming to make available service contracts, which serve to specify the communication form among software applications.

SOA allows the automation of business processes related to the financial and accountancy areas, through the creation of information systems (SOA applications). These systems, due to the flexibility, interoperability and alignment with business processes necessary to SOA applications, allow significant improvements to be made to the enhancement implantation in business processes, due to the facility to change and modify these systems. SOA is a change in the structure of an environment of software development and maintenance. There is no predefined SOA tool or framework; the SOA environment must be constructed according to the organization's characteristics, through an incremental and iterative approach. For its implementation, a correct governance approach is necessary. Central staff with the responsibility for deciding on the general aspects of the SOA in the corporation must exist. On the other hand, one of the key elements for its success is decentralization and the delegation of attributions and responsibilities. Therefore, it is important to find a balance between centralization and decentralization.

SOA helps in the reduction of the conceptual gap among business and IT areas. This approach's (SOA) goal is to structure distributed systems based on the abstraction of rules and business functions. This abstraction guarantees that information systems, although having technical characteristics, are projected in order to be understood by people from business areas, also generating, through the flexibility and connectivity inherent to SOA applications, better service adaptation to the changes in the requirements demanded by the business. 
Concepts like scalability and flexibility gain force in the SOA architecture, which allows its uses in the implementation and growth of the organization's systems. The way to guarantee flexibility in corporative systems is to support heterogeneity, decentralization and even tolerance to failures (Josuttis, 2007). The aspects of scalability and the low coupling directly influence the failure tolerance issue. The lower the coupling, the smaller the dependence among systems and, thus, the smaller will be the consequences of failure occurrence in other systems.

It is common to find in the literature the imprecise idea that SOA and web services are synonyms. SOA is a paradigm, an architecture, while web services are SOA architecture components (Josuttis, 2007). A web service represents the materialization of the idea of a service that is made available on the Web. These web services represent the business logic that allows clients to send requests and receive responses.

A web service provides an infrastructure uncoupled to a platform, which makes its development and integration with different technologies easier. Due to possessing a low-coupled internal structure, web services allow services to change without affecting their usage by the client, therefore constructing modulated systems that achieve more adequate levels of integration. The web services must be seen as a convergence of various technologies, like HTTP (Hyper Text Transfer Protocol) (HTTP, 2008), XML (eXtensible Markup Language) (XML, 2006), SOAP (Simple Object Access Protocol) (SOAP, 2007), WSDL (Web Service Description Language) (WSDL, 2007) and UDDI (Universal Description, Discovery and Integration) (UDDI, 2004). A relevant technology for integrating web services with the XBRL language is the SOAP protocol. SOAP is a specification for exchanging information structured in distributed environments, the main goal of which is to ensure interoperability among different systems, through the usage of the XML and HTTP protocols. The XML language is used to create messages that will be exchanged among the distributed applications. The HTTP protocol acts as a means for transmitting these messages, besides providing a structure for remote procedures' calls as a pattern of interoperability. Messages or SOAP files, responsible for packing the content of client requests and responses, consists of four elements in their composition: the envelope, the header, the body and the fault: 
- $\quad$ The envelope element is a mandatory component of the structure of SOAP messages. It defines the SOAP message's root and determines how the XML file, regarding the request content, is transformed into a SOAP message and how this message must be translated by web services in the service;

- The header element is an optional component of SOAP messages that, when present, must appear immediately after the envelope element. The header must contain SOAP message specific information, generally information regarding the web service that will receive the message, for example, security information;

- The body element is a mandatory element that stores the file to be transmitted (the XML file, which contains the SOAP message content) and, optionally, a failure definition (related to the fault element) that may occur;

- The fault element is the component of the SOAP message's structure that is responsible for capturing the errors occurring in the web services and, optionally, defines messages of error that may occur in files' transmission and reception. In the case of being present in the structure of SOAP messages, the fault element must be inside the body element and must be unique.

The XML file transformation process, which contains the content of requests in a SOAP message, consists of the following steps: (i) first, the XML file is packed (SOAP message content) into the body; (ii) next, the packing of the body element inside the envelope element occurs; (iii) optionally, the header element is attached to the message; (iv) optionally, the failure (fault) block is attached to the message; (v) finally, the SOAP message is transmitted.

\section{XBRL (EXTENSIBLE BUSINESS REPORTING LANGUAGE)}

Aiming to improve the quality and reliability of financial reports' emission, XBRL provides a necessary structure to perform data and financial information interchange in a standardized way among different information systems. Although the use of XBRL has expanded to other non-financial areas, its widespread use remains for the exchange and disclosure of financial data. Through its structure, XBRL contributes to the resolution of problems related to financial information data format diversity, because this information is located in different data systems and formats, which causes a large amount of data manipulation, to promote this information's consolidation in the financial report (Silva, 2003). 
XBRL is a language based on XML (Extensible Markup Language), which was developed for the representation and interchange of financial information. Aligned with the same opinion are Silva et al., (2005), who complement it by affirming that XBRL provides data structuring for financial reports, performing the automatized interchange of these reports among independent information systems. Therefore, XBRL becomes a standard to represent and interchange financial information reports, including accounting demonstrations and information for regulatory government agencies and for investors.

XBRL enables the creation of a standardized vocabulary for interchanging financial information reports, making it possible for companies to create different types of financial reports, e.g. financial statements, reports for supervisory and regulatory agencies, risks reports and credit line reports. It is important to emphasize that XBRL allows companies to codify their financial statements according to the demands and regulations imposed by regulatory agencies and laws, thus ensuring that the financial information provided and attested by the CFO (Chief Financial Officer) is precise and reliable.

The XBRL data exchange is based on three types of XML files, which form a financial report representation: (i) an XML Schema (XML Schema, 2004) file containing the definitions of the elements used in the XBRL instance file; (ii) Linkbases files based on XLink (XLINK, 2001), expressing the relationships among the elements defined in the previous file; and (iii) an XBRL instance file, the report itself, which contains the financial facts with the data to be reported. The Linkbases and XML Schema file constitute the financial report taxonomy and the XBRL instance file is the report itself.

The process of emitting a financial report through XBRL comprehends the following: (i) defining or selecting the taxonomy, which must address the company's needs and/or the requirements of the regulatory agencies to which the company will provide the financial information; (ii) defining the XBRL file instances, composed of the data that were extracted from their databases. In this context, two points deserve to be highlighted. The first refers to the aspect of taxonomy creation, which must consider which financial facts one wishes to express and the hierarchy among these facts. The second regards the creation of the XBRL file, which may capture the financial data to form a database and must be validated on the taxonomy, that is, it must be in conformity with what was 
defined in the taxonomy; (iii) making available the XBRL instance file or interchanging among the systems and databases. It is also possible to use resources like XSLT (Extensible Style Sheets Language Transformation) (XSLT, 1999) or an extension of XBRL 2.1 specification, named iXBRL (IXBRL, 2010), to present an XBRL report in other formats (e.g. HTML and PDF), to make the reading more pleasant for the user.

\section{APPROACHES AMONG SOA AND XBRL}

This section discusses the works related to SOA and XBRL technologies. These works are associated with information systems' flexibility and financial reports' emission.

\subsection{XBRL and the Growing Use of Online Filing and Electronic Payments}

Information technology has been evolving at a velocity that is hard to follow and the competitive pressure has been forcing innovation within organizations. The work of Ascenção et al. (2006) aims to demonstrate that for a company to be competitive, in the globalized world, it must adopt a new attitude and adhere to new technologies, among them XBRL, to allow better flexibility, agility and efficiency for the managers, who can start to evaluate better the diverse decisions to be made. In order to achieve this, the authors discuss the reasons that lead to the adoption of XBRL, its functioning system and the benefits generated by the adoption of this language for the emission of financial reports. The authors claim that XBRL facilitates the building and disclosure of financial reports.

The work by HM Revenues \& Customs - UK Government (2009) highlights that XBRL implementation for online filing is on course, through a technical subgroup from XBRL International (XBRL, 2006), to develop solutions that allow companies' accounts and tax calculations to be filed online, in XBRL format. The author affirms that many benefits exist that have not yet been quantified but that justify XBRL implementation to perform online filing and electronic payment. The benefits are hard to quantify; however, the author expects them to be higher than the costs, thus providing a real benefit for companies. These benefits include: (i) a lower error possibility; (ii) faster data transmission; (iii) immediate recognition of received data; (iv) use facility; (v) enhancement of reliability and security for electronic payments; and (vi) improvement in data communication and transfer among companies' different departments and offices. 
However, in spite of the work by Ascensão et al. (2006) having shown XBRL's relevance to the building and disclosure of financial reports and the work by HM Revenues \& Customs - UK Government (2009) having laid the foundation for the decision regarding the use of XBRL, these works do not cover the integration between XBRL and SOA, nor do they approach aspects associated with flexibility and alignment with business processes, as the information systems based on XBRL have to adapt to the modifications that occur in financial report requirements, e.g. legal and regulatory.

\subsection{The Importance of Business Processes and IT Governance for SOA Governance}

SOA governance represents a subset of elements that compose IT governance and have as a goal to regulate the management of the processes and activities that compose the life cycle of solutions oriented to service, through the definition of rules, policies, actions and responsibility maps inside the organization (Marzullo, 2009). According to the author, SOA governance features as a technique to help the organization to implement control mechanisms more efficiently and securely, focusing on the development of solutions oriented to services. Marzullo (2009) emphasizes that the creation of an SOA competence centre must be considered, which aims to help in the performance of the work of the infrastructure, architecture and processes. Among the SOA governance life cycle activities, those that deserve to be highlighted are: (i) strategy definition activities, the goal of which is to establish the strategic direction, identifying and prioritizing business processes and IT resources that will be used in services' construction; (ii) change management and version control, which aims to manage the changes and versions of the services, thus allowing their evolution in a controlled way; and (iii) security activity, which due to the high levels of vulnerability that the architectures oriented to service present, aim to guarantee satisfactory security levels in the business contexts that are sensitive to threats (Marzullo, 2009).

SOA is a paradigm for conceiving and implementing business partnerships (inside and outside organizational borders), being of interest to software suppliers and researchers (Aalst et al., 2007). In this paradigm, Aalst et al. (2007) emphasize that the functionality offered by business applications is encapsulated inside web services that may be invoked by applications or other services, through a subset of Internet patterns, including HTTP, XML, SOAP, WSDL and UDDI 
(Aalst et al., 2007). The authors also explain that once the web services provided by different existent organizations are implemented, the aim is to implement business collaborations, thus creating composed web services.

Marzullo (2009) highlights the importance of IT governance for SOA governance. Aalst et al. (2007), however, affirm that web services are composed to support and provide flexibility to business processes, making them more adaptable to the changes that occur in business requirements, concluding that the relation between business process management and service-oriented architectures is highly relevant (Aalst et al., 2007). Both works have contributed to the foundations and construction of the model proposed in this article.

\subsection{The Impact of SOA on Entrepreneurial Systems, Organizational Structures and Implantation Phases}

A company must be flexible and agile in order to attend to the changes related to its needs and operate in the variable environment of a global economy (Bieberstein et al., 2010). To do so, the authors show that it is necessary to align the entrepreneurial requirements with the information technology, through the service-oriented architecture (SOA). However, in order to be efficient, the SOA paradigm also needs to rupture the barriers related to the organization behavioural practices. Therefore, the authors show the SOA corporative challenges (e.g. cultural changes), as well as the need for redrawing the existent organizational models, optimizing the workforce and the interaction of business processes among departments to leverage the new IT systems. The authors conclude that, in order for a company to obtain SOA value, a cultural and organizational transformation is essential. However, although this change is always accompanied by uncertainty, according to the authors, the potential benefits (flexibility and agility to attend to changes) overcome the risks (uncertainties).

Organizations vary a great deal in their goals, scope, size and structure (Brauer and Kline, 2005). However, a careful study of their needs regarding information technologies leads to the identification of a wide set of common concerns. According to the authors, a company dedicates almost $80 \%$ of its budget to maintaining the existent systems. The main reason for this high cost is the inflexible nature of the applications that are projected. The authors also show that companies' executives demand increasingly adaptable IT applications. According to Brauer and Kline (2005), research proves that nearly $90 \%$ of the world's 
companies cite speed, flexibility and adaptability as priorities (Brauer and Kline, 2005). Therefore, the authors conclude that the ideal way to fulfil these business requirements (common concerns and requirements) is what they call an adaptable company. An adaptable company is an organizational view in which the business and IT are synchronized to capitalize on change. Being an adaptable company helps to reduce IT costs, making the IT more flexible to deliver what the business really needs: simplicity, agility and value. According to the authors, an adaptable company allows a strong linkage to be reached between business and IT, enabling the company to respond quickly and easily to changes and helping to maximize the response, mitigate risks, improve performance and increase agility.

A script to perform SOA implementation in medium and large-size corporations is described by Waldman (2009). The author suggests that the implementation must be conducted in phases, starting with a single system that is small and simple. Following the success of this first phase, the same process is repeated for other systems, until it is performed for the whole organization. If the evaluation of the first phase is not satisfactory, new attempts must be carried out until the organization is familiar and confident with the new concept (SOA) and, therefore, may advance to other systems.

In the last years, SOA, together with information systems, has achieved greater recognition as an alternative to develop information systems (Niemann et al., 2008). In the context of SOA, governance represents a new challenge. Currently, there is a series of different approaches to SOA governance frameworks, which differ widely in scope and capacity. According to the authors, most of these frameworks are oriented towards products and, consequently, developed by the software industry (e.g. HP, IBM, Microsoft). Niemann et al. (2008) conclude by presenting an approach to an SOA governance generic model. This model integrates an efficient control cycle with the concept of a maturity model in SOA. The authors report that the SOA governance generic model is based on COBIT (Control Objectives for Information and related Technology) good practices and that it provides a common set of IT metrics. This model represents a fundamental and generic approach to SOA governance.

SOA governance is essential to realize the benefits of service-oriented architecture (SOA), because, without efficient SOA governance, organizations face significant challenges, including: (i) difficulties in the conception of 
decision-efficient structures; (ii) a lack of financing for SOA services; (iii) a lack of consistent governance processes; and (iv) a lack of alignment between IT and business areas (Hojaji and Shirazi, 2010). According to the authors, studies performed on existent SOA governance frameworks show that these structures do not document the SOA governance processes completely, together with the procedures. These frameworks are also not expressive enough to cover all of the important elements of SOA governance. Therefore, this work presents an SOA governance framework that has a life cycle formed by COBIT governance good practices, together with service management activities. According to Hojaji and Shirazi (2010), COBIT was used due to its mature and efficient IT governance structure, which also approaches IT process evaluation, and may be used to describe the processes, targets and goals of activity control in the IT area.

The lack of governance mechanisms is frequently seen as the main reason for SOA project failures (Ott et al., 2010). In this work, the authors present some elements that can orient organizations in the implementation of an SOA governance approach. To achieve this, a mapping practice of IT governance, COBIT and ITIL (Information Technology Infrastructure Library) to the SOA domain is presented. These authors focus on the changes that are necessary to ITIL and COBIT IT governance approaches, with the objective of maximizing the business potential of the service-oriented architecture. The work enables organizations to compare their own IT governance model with roles and activities, aligning it with the SOA approach. This allows them to identify divergences and weak aspects in the IT governance approach and make it adequate for orientation to services.

Although the work of Bieberstein et al. (2010) highlights the importance of SOA to provide organizations with agility and flexibility and the work of Brauer and Kline et al. (2005) proves the need companies have to possess an IT environment that is fast, flexible and adaptable, besides the work of Waldman (2010), which has contributed, through its script of implementation, to the construction of this paper's SOA implementation model, they did not approach the aspects related to the integration of SOA applications with XBRL, to facilitate an improvement in the quality and reliability of the emission of financial reports, as they did not have this purpose. The same can be observed in the works of Hojaji et al. (2010), Niemann et al. (2010) and Ott et al. (2010). 


\subsection{IT Innovative Traces in Service of Controllership}

The complexity of contemporary entrepreneurial processes linked to accounting and information technology areas indicates that the stock market, the controllership departments and the regulatory agencies, like the US-SEC and central banks, are gradually demanding and orienting companies to adopt a new technologic-operational dynamic that is able to attend rigorously to the informational demands of organizations (Biancolino and Cristofoli, 2008). At the same time, applications' diversity and items related to the IT operational infrastructure are continually increasing, leading to a deadlock in obtaining greater informational efficiency in this area due to its complexity.

Accordingly, the authors performed an analysis of SOA and XBRL technologies, which constitute an important resource that may be used by controllerships, in order to offer an IT solution that would provide a channel through the business demands and regulatory agencies. Through the analysis performed, the authors concluded that SOA is a facilitator for developing corporative information systems as modular business services that may be integrated and easily reused, creating a flexible and adaptable IT infrastructure. The analysis regarding XBRL technology, however, demonstrated the importance of this language for the business area, due to the facility offered for sharing and searching financial and business information in the context of organizations' information systems.

Although the authors highlight the importance of these technologies for the accounting area and for the emission of financial reports, in order for the development of information systems through SOA and XBRL to become successful, it is also necessary to have a structure of business process management, which will be automatized through SOA. Besides that, the work does not present a model for implementing SOA integrated with XBRL through a web service.

\subsection{An Integrated Solution of Automated Reports in Basel II}

Gluchowski and Pastwa (2007) propose to investigate ways of performing the interchange of financial and business data. Usually the financial report emission process is characterized by the use of different data formats representing the output of different information systems, necessitating data transformation from 
one format to another. The authors emphasize that this manipulation may generate losses, corruption and incompatibility of data, which may lead to negative impacts on the organization's business. The authors discuss issues related to financial information interchange, in a reliable and fast way, through SOA technologies, with web services and financial reports, therefore also making the business processes and the systems more flexible. Gluchowski and Pastwa present a proposal for developing financial information systems, through SOA with web services and XBRL, and discuss the benefits that SOA services would bring to financial reports' emission (Gluchowski and Pastwa, 2007). However, despite this work having set the foundations for this paper, it does not present a model for SOA implementation and integration with XBRL.

\subsection{Continuous Audit based on XBRL and SOA}

Relying on XML and SOA, Chen and Sun (2007) present a model for continuous auditing. According to the authors, one of the benefits of using SOA web services to assist with continuous auditing is that each company does not have to make great efforts to study the format of XML documents and build applications by itself to convert data. In addition, through selecting appropriate XML transformation and security mechanisms, companies will not be afraid that others will disclose their confidential data. The proposed model will be helpful for implementing a collaborative continuous auditing system among audit firms, client companies and third parties. The purpose of this paper is to present a collaborative continuous auditing model that employs the emerging XML technologies, like XBRL, in an SOA environment to support continuous auditing with clients' ERP systems.

As globalization intensifies throughout the world, the need for accurate, reliable and real-time financial information by companies, regulators and other users has also intensified. Therefore, is necessary to provide real-time assurances to decision makers. According to Qiushi et al. (2012), some of the current challenges of computer auditing are the obstacles to retrieving, converting and translating data from different database schema. Thus, they propose a collaborative computer auditing system under an SOA-based conceptual model. By utilizing the XBRL document standards and data transformation applications developed by different companies and software venders, the authors propose to wrap these application as web services that will be easy implemented in an SOA 
environment. The authors affirm that under the SOA environments, the multiagency mechanism will help the maturity and popularity of data assurance services over the Internet. By the wrapping of data transformation components with heterogeneous databases or platforms, it will create new component markets composed of many software vendors and assurance service companies to provide data assurance services for audit firms, regulators or third parties.

In order to solve the problems of the accuracy of the data, the real-time and comprehensiveness of the audit and the flexibility of the audit, an SOA-based conceptual model for continuous auditing is proposed by Ye et al., 2008. Two models of SOA (a service registry model and an enterprise service bus model) are applied to this conceptual model. The model shows how the new technology using XBRL, a Just-In-Time database and the Intelligent Agent can help effectively audit the transactions between the client and the third parties. According to the authors, the main advantages of SOA are alignment of IT with the business, maximal reuse of IT assets and IT infrastructure flexibility.

Although the authors (Chen and Sun, 2007; Ye et al., 2008; Qiushi et al., 2012) do not discuss how to perform the integration of XBRL and SOA for the aspects related to governance and management, these studies reinforce the need for a management model to integrate these technologies.

Therefore, through the analysis of correlated works, it is possible to conclude that no proposal can be found in the literature that presents SOA and XBRL integration in a detailed way. For this reason, in Section 5 of this paper, a model for the management of SOA implementation and integration with XBRL is presented and discussed. This model has the purpose of facilitating and improving the efficiency of business processes of financial reports' issue, thus providing an improvement in the quality and reliability of these reports.

\section{MODEL OF SOA IMPLEMENTATION AND INTEGRATION WITH XBRL}

The model of SOA implementation and integration, through web services, with XBRL aims to provide information systems that are more flexible, interoperable and aligned with the business processes, providing a better facility for adaptation to changes that happen in the organization's business, besides providing the integration of these services with XBRL. To provide better comprehension and 
organization, the model is divided into three phases. The phases, however, involve activities that may be subdivided into tasks.

This model considers that the following actions must be taken in order to achieve a satisfactory performance: (i) start SOA implementation with a single system, which is small and simple, preferably a system that enables good migration; (ii) repeat the same process for the other systems, until it is performed for the whole organization; and (iii) perform service composition instead of developing new information systems. Figure 1 illustrates the organization of the model. Its phases are organizational needs' identification, implementation and implantation, which will be discussed in the following sections.

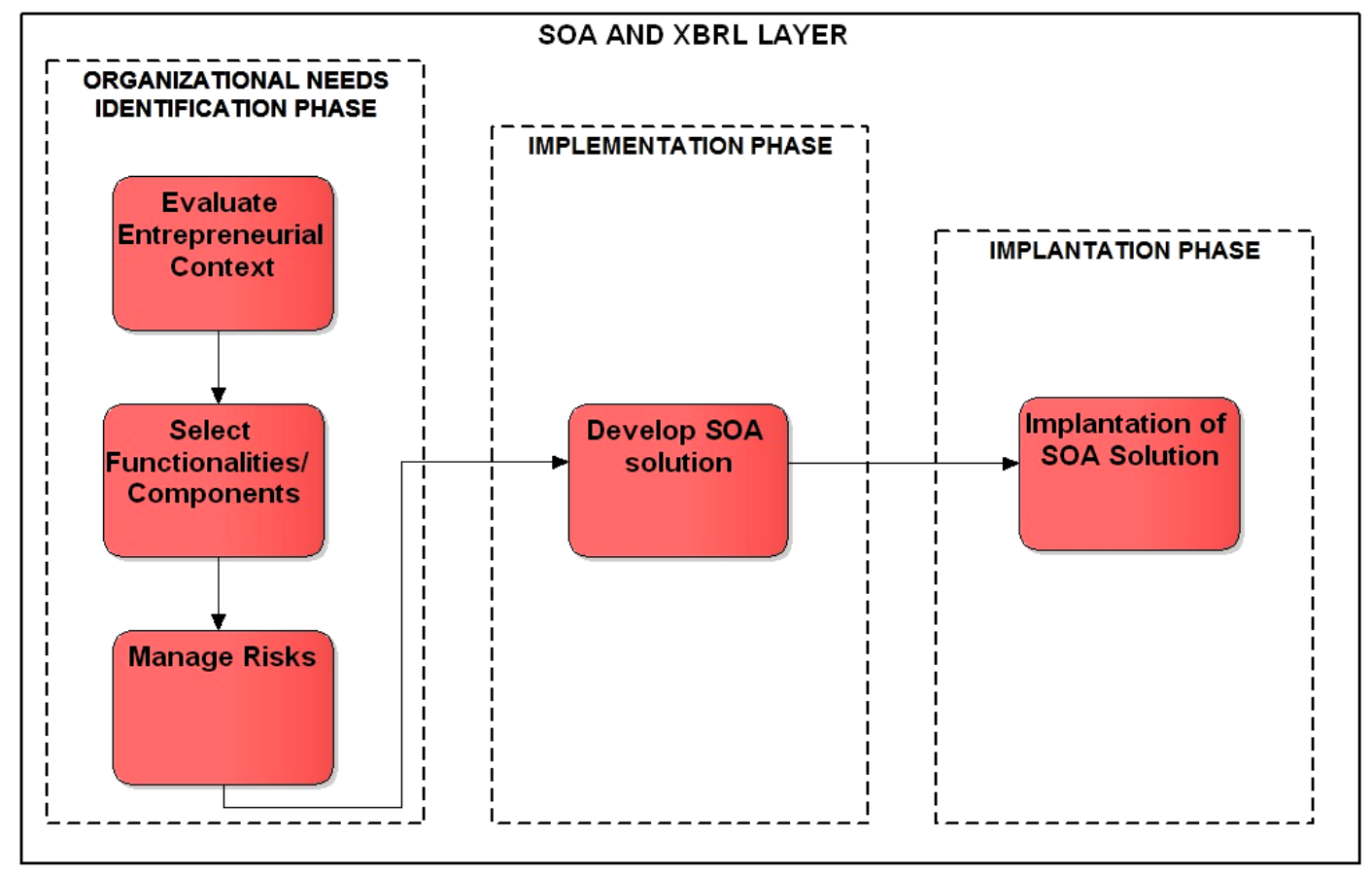

Figure 1: Model for SOA implantation and integration with XBRL

\subsection{Organizational Needs Identification Phase}

This phase aims to evaluate the current context of the organization, besides identifying the elements that will become SOA services and provide risk management. To do so, this phase contains the following activities: 


\subsubsection{Evaluate the Entrepreneurial Context}

This activity aims to evaluate the current scenario of the company, analysing the following elements: (a) the business processes, which must already be structured and defined; (b) the information systems, which automatize the business processes and from which will be extracted the functionalities that will be transformed into services; and (c) the IT governance model, necessary to structure and control the IT processes, which will allow the adequate use of resources for service-oriented solutions development and maintenance. The input and output related to this activity are presented in Table 1.

\begin{tabular}{|l|l|}
\hline Input & Output \\
\hline Business Processes & \multirow{2}{*}{ Current Scenario Analysis Report } \\
\hline Information Systems & \\
\cline { 1 - 1 } IT Governance Model & \\
\hline
\end{tabular}

Table 1: Activity "evaluate entrepreneurial context" - input and output

\subsubsection{Select Functionalities/Components}

This activity produces a selection of components or functionalities from the information systems that will become services. The inputs and outputs related to this activity are shown in Table 2.

\begin{tabular}{|l|l|}
\hline Input & Output \\
\hline Business Processes & $\begin{array}{l}\text { Relation of the Components and/or Functionalities } \\
\text { that Will Become Services }\end{array}$ \\
\hline Information Systems & \\
\hline
\end{tabular}

Table 2 - Activity "select functionality/component" - input and output

\subsubsection{Manage Risks}

This activity aims to perform the risk management in SOA implementation. This activity is divided into two tasks: (i) identify and evaluate risks in SOA implementation; and (ii) control risks in SOA implementation. These tasks are detailed below: 


\section{(i) Identify and Evaluate Risks in SOA Implementation}

This task identifies and evaluates the risks associated with SOA implementation. The SOA adoption process is susceptible to some occurrences (risks) that may be decisive for its conception and must be identified, because they may make the SOA implementation unviable in the organization.

It is necessary to understand how the company management deals with issues regarding risks for identifying the company profile of risk treatment, since this may vary in each company, ranging from more careful profiles to more aggressive profiles. In this way, evaluating files related to incidents and the risk management policy adopted by the company may help in the identification of the company risk profile. Once this profile is identified, the risks of SOA implementation must be identified. The risk identification in the SOA implementation process must evaluate: (i) whether the company's technical staff understand the SOA and have the technical capacity for its adoption; (ii) the functionalities that will be transformed in SOA services; (iii) the company's capacity to perform the necessary changes for realizing the benefits of SOA implementation; (iv) the existence of IT governance and senior management support; (v) the business process management methodology used and whether there are well-structured and efficient business processes; (vi) the budget allocated to SOA adoption; and (vii) the adequacy of the methodologies and practices of risk management. Having identified the risks, how they will be treated must be defined.

It is important to document how the risk management will be managed and register all of the risks identified during this activity and throughout the SOA implementation. The steps to execute this task successfully are: (i) identify the risks related to the services offered by the company; (ii) identify the risks related to the new developments and activities that will be performed in SOA implementation; and (iii) define the treatment that will be given to the risks found.

\section{(ii) Control Risks in SOA Implementation}

This task aims to provide the necessary subsidy to the company to perform risk control in SOA implementation. The IT governance implementation, through the use of good practices from COBIT and ITIL and business process management, by business process management (BPM) good practices, helps in understanding, structuring, maintaining and evolving the processes (IT and business), besides 
ensuring a commitment from the high administration. The uses of these good practices contribute to controlling risk and threat that may emerge in SOA implementation.

Companies wishing to prevent and control the various factors (organizational or technical) that generate risks to the SOA adoption process should gain a unique understanding of their business and IT processes and should have the full support of the senior management, thus ensuring the support of all the departments that are involved in the initiative. The company must also promote and diffuse all the information needed in order for the SOA implantation process to take place in an acceptable way. The outputs and inputs related to this activity are shown in Table 3.

\begin{tabular}{|l|l|}
\hline Input & Output \\
\hline $\begin{array}{l}\text { Company Current Scenario Analysis } \\
\text { Report }\end{array}$ & $\begin{array}{l}\text { SOA Implantation Risk Management } \\
\text { Plan }\end{array}$ \\
\hline $\begin{array}{l}\text { Relation of the Components and/or } \\
\text { Functionalities that Will Become } \\
\text { Services }\end{array}$ & Risk List \\
\cline { 2 - 2 } & Plan of Response to Risks \\
\hline
\end{tabular}

Table 3: Activity "manage risks" - input and output

\subsection{Implementation Phase}

In this phase, the development of the SOA solution that will attend and will be in conformity with the organization's needs is proposed. Therefore, this phase contains the following activities.

\subsubsection{Develop the SOA Solution and Integrate with XBRL}

This activity is related to the implementation of a life cycle in the development of software, composed of the requirement survey activities, analysis, project, services implementation and services. It is relevant to highlight that in the analysis and project the aspects related to the reuse and integration of the services that will compose the SOA solution must be considered.

The web services represent a way of implementing services in SOA and provide an infrastructure independent of the platform, making the development and integration of different technologies easier. This activity also has as a goal to 
integrate the web services with XBRL. Accordingly, an architectural model that illustrates this integration is presented.

The architectural model of integration may be seen in Figure 2, which shows how the integration between XBRL and web services may occur. It is possible to observe in the architectural model that the web services serve both to format the data with XBRL and to consolidate them in financial reports (Borges and Silva, 2012). Firstly, it is necessary to transform in web services the system functionalities that are responsible for the information that will be present in the financial reports to be emitted. It it thus necessary for the web services to be able to extract data from the systems, putting them into XBRL format, so that later, through this data consolidation, it is possible to create a financial report, thus performing the integration between the two technologies. An issue to be considered in this architectural model is that the business processes are the elements of the architectural model that drive, through the laws and regulations that they are linked to, the needs of the XBRL documents, which will be built by the organization through its web services.

XBRL integration with web services occurs when the XBRL instance document is packed inside the SOAP message that will be transmitted. It happens after the definition or selection of the XBRL taxonomy and the later creation of the XBRL instance document, according to the organization's needs. It is important to highlight that the taxonomy defined must serve as a standard, because the XBRL document must be validated through it during its creation and after the unpacking of the SOAP message, with its receiving (e.g. by the regulatory bodies or company main office) for processing. The inputs and outputs related to this activity are displayed in Table 4.

\begin{tabular}{|l|l|}
\hline Input & Output \\
\hline $\begin{array}{l}\text { Company Current Scenario Analysis } \\
\text { Report }\end{array}$ & \multirow{2}{*}{ Web Services Developed and } \\
$\begin{array}{l}\text { List of the Components and/or } \\
\text { Functionalities that Will Become } \\
\text { Services }\end{array}$ & \\
\hline
\end{tabular}

Table 4: Activity “develop SOA solution" - input and output 


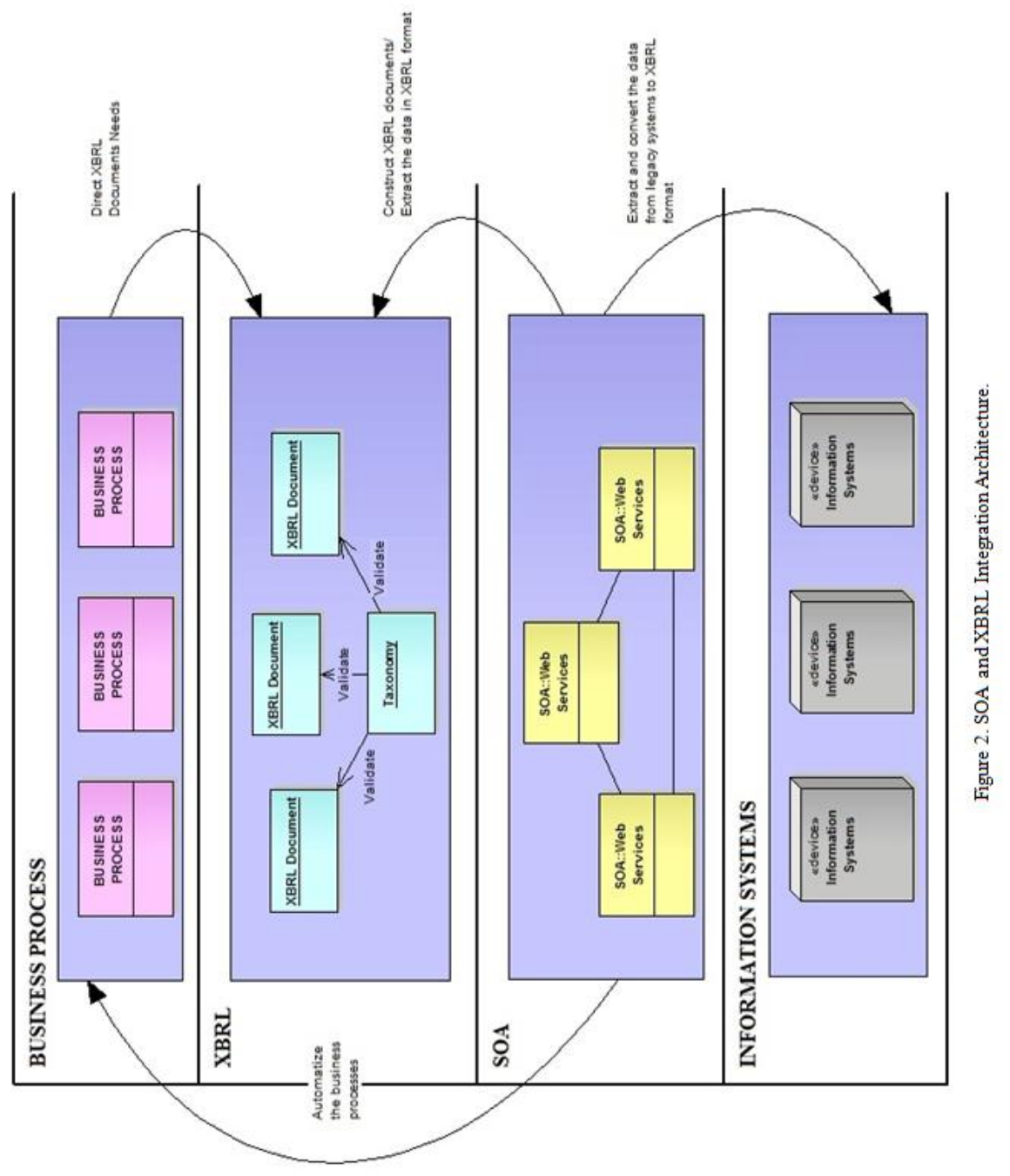




\subsection{Implantation Phase}

This phase is responsible for putting into operation the services developed in the previous phase. If the first attempt at SOA implantation is not satisfactory, the company must perform an analysis of the reasons leading to the lack of success, so that the errors made and the risks that were concretized during the implantation attempt may be properly corrected or eliminated. Subsequently, when the organization is more familiar with and confident about the concept (SOA), new tries may be executed. There is also the possibility that the failure (of SOA implantation) is related to other factors, neglected in the risk identification and analysis (Section 5.1.3 item (i)); in this case, the staff responsible for SOA implantation must perform a critical evaluation to identify which factors led the SOA implementation to a negative outcome. After the first implantation success, the organization must extend its implementation to other systems. However, until the end of SOA implantation, the information systems (legacy systems) and SOA applications will coexist concomitantly. During the process of adopting SOA, the governance policies in the IT area and business processes must be totally implanted.

With the maturity of the organization, regarding SOA use, it is expected that new projects will become faster in their implantation, less expensive and involving fewer implantation problems, because the higher the level of reuse, the lower the effort for developing and the chance of problems occurring. When all the systems are oriented towards service, the organization must have a stable environment and the services must be in the company services repository, without the presence of duplicated services. In this context, it is important for any new service to follow the development process and the organizational policies directed to the SOA. The reuse of the services, made available by the company, must also be conducted through the service repository and with a formal contract of service provision (Waldman, 2009).

\section{AN SOA AND XBRL INTEGRATION SAMPLE}

To show the use of the proposed model a scenario was built to illustrate an SOA solution that considers the aspects of reuse and service integration in service development. Decomposing the business process in web services is considered the top-down approach. To exemplify the transformation of the business process in the SOA service, an example was created of how the solution development 
activity must be conducted in the company. Figure 3 shows the partial decomposition of the financial business process in web services. It is possible to observe, through this example of use, the transformation into web services of the tasks that compose the activities "maintain client data" and "issue and transfer reports", which belong to the subprocess "accounts to receive", which is part of the process "manage financial resources". Through this decomposition, it is possible to transform the business process in tasks later to develop the serviceoriented solution, thus automatizing the execution of its tasks and activities.

It is important to highlight that the complete automation of a process or subprocess, for example, "accounts to receive", is a result of a service composition that groups services into modules, creating functionalities that are semantically equivalent to the activities that compose the business process. After that, these modules are orchestrated according to the rules of business and process execution flow. The composition of web services, using the orchestration technique (Erl, 2009), must reflect a collaboration environment among the web services that is dynamic, flexible and adaptable to the constant changes that occur in the business processes. To implement the web services orchestration, the language WS-BPEL - Web Services Business Process Execution Language must be used, which is the main technology used to express the web services' composition logic (Erl, 2009).

To illustrate the integration process of the web services with the XBRL language, a second scenario was constructed. This scenario of integration between XBRL and SOA, which may be visualized in Figure 4, is composed of three branches of the company, which possess different information systems. These systems were built with the NET, PHP and Delphi programming languages and use the SQL Server, MySQL and Firebird data basis, respectively. These systems send their financial information in XBRL format, through web services, to their main office, which then consolidates the information into an integrated financial report to send later to regulatory agencies. The company's main office information system was developed with the Java programming language, using the Oracle database. 


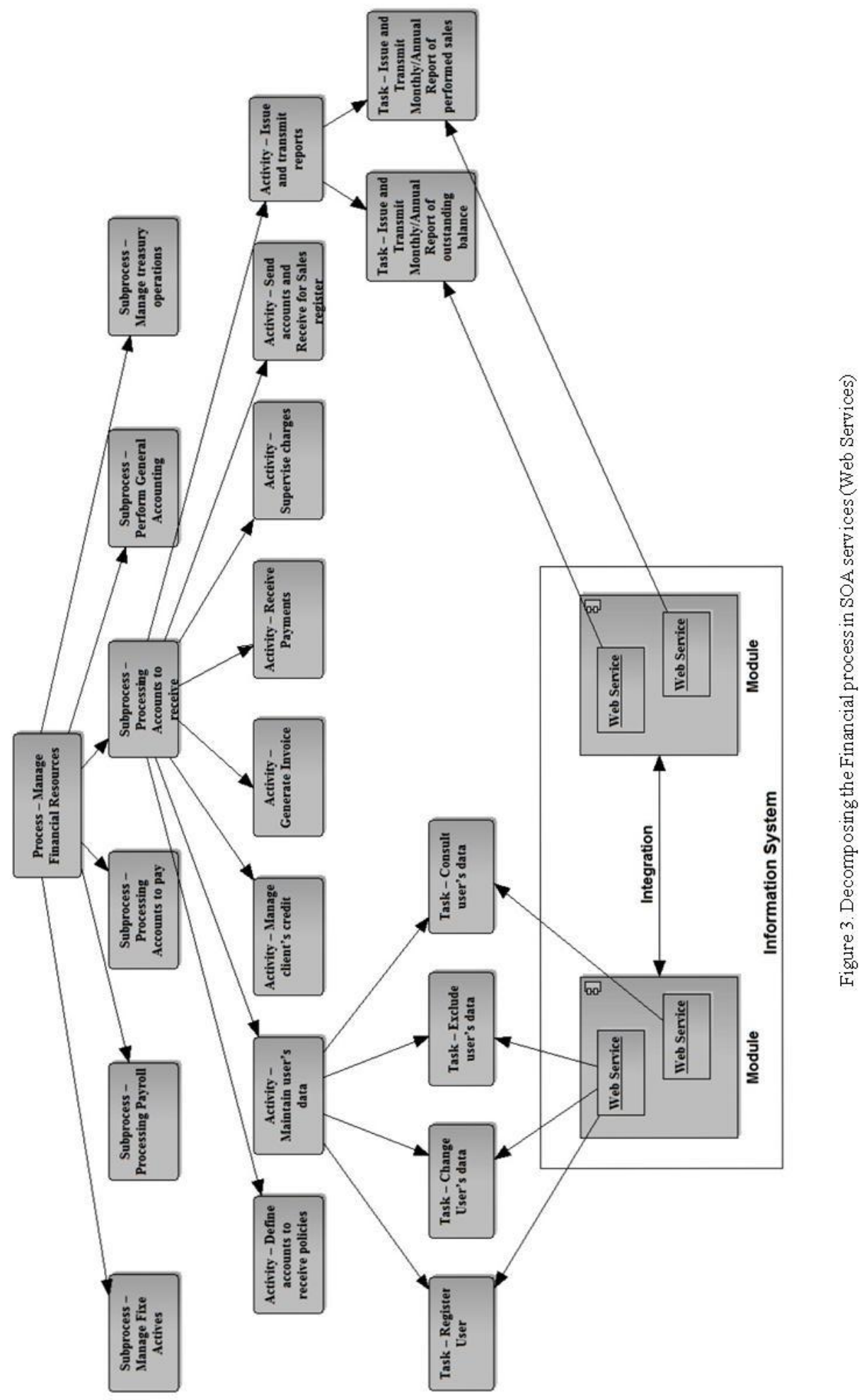




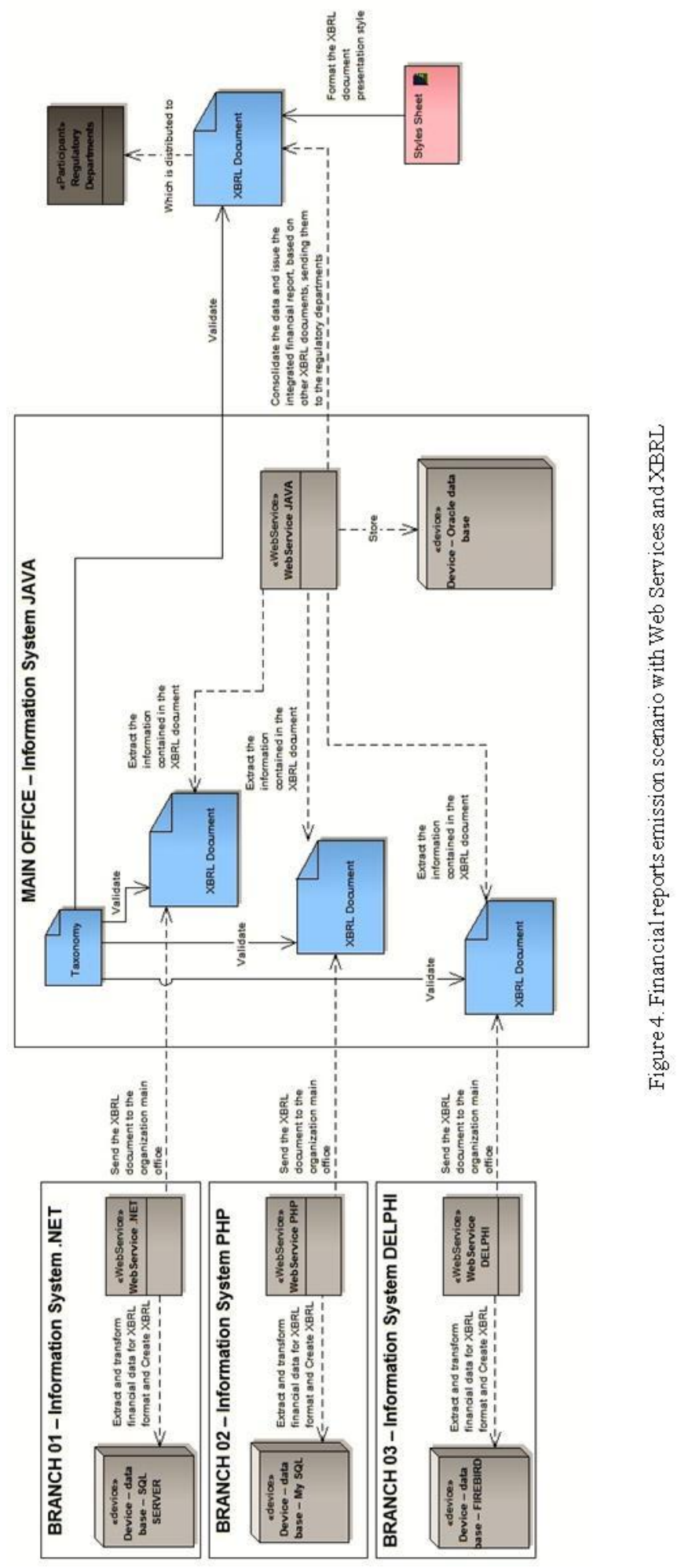


In this context, XBRL integration with web services can take place at three different moments. The first moment occurs in the creation of the XBRL document, when the web services access the heterogenic data basis to extract the data and transform them to XBRL format, according to the rules defined in the taxonomy, to generate the financial report. The second moment occurs when the company branches use the web services to send the XBRL document that represents the financial report of a given month or year to their main office, so that the reports can be integrated into a single report. The third moment occurs when the main office makes available a web service to consolidate the information that is in XBRL format and that was obtained from the branches, sending, later, the XBRL document, which represents the integrated financial report of a month or year, to the regulatory agencies.

\section{CONCLUSION}

Aiming to provide an organized and adaptable environment, this article proposes an implementation model of SOA and integration with XBRL. The model proposed in this paper contributes to the elucidation of problems related to the diversity of the data format of financial reporting and to improve the alignment and adaptation of business processes to the strategic needs of the organization, through the greater flexibility of information systems to the changes that occur in business requirements. This model also provides better interoperability between systems and improves the quality and reliability of financial reporting.

With the use of XBRL, this model will be able to contribute to decreasing the need for financial data format transformation and to decreasing the data extraction cost of financial reports. The risk of error and, consequently, an increase in financial information reliability are also reached, due to the decrease in the need for efforts to transform and extract data of different formats.

With the use of service-oriented architecture (SOA), this model can automate the business processes related to the financial and accounting organization, making these systems more flexible to changes and aligned with business processes that promote financial reporting.

With the conclusion of this work, it was noticed that some issues remain open, which can be analysed in order to enlarge the set of contributions available. Accordingly, some directions for future work are listed below: 
(i) Methodology: During the model building, the need to establish a methodology was observed. This methodology should record in a standardized way all the information related to the tasks and activities included in the model layers, allowing the unambiguous documentation of the model of governance and management of IT. Studies are being conducted to implement this methodology.

(ii) Business information systems: During this work, we realized the possibility of adapting the proposed model to the development of business intelligence (BI) systems. These systems would facilitate a better analysis of the performance of processes and hence the company strategy. Studies should be conducted to define the best form of model adaptation to the development of these BI systems.

\section{REFERENCES}

AALST, W.M.P. VAN DER; LEYMANN, F.; REISIG, W. (2007): "The role of business processes in service oriented architectures", International Journal of Business Process Integration and Management, vol. 2, n. 2: 75-80.

ASCENÇÃO, H.S.; SOUZA, I.B.; SOUZA, E.A.F. (2006): “XBRL, Paradoxo para o Mercado globalizado". http://bit.ly/1jw9c8V

BIANCOLINO, C.A.; CRISTOFOLI, F. (2008): "SOA e XBRL: Traços inovadores de TI a serviço da Controladoria”, paper presented at the 5th International Conference on Information Systems and Technology Management, São Paulo, Brazil.

BIEBERSTEIN, N.; BOSE, S.; WALKER, L.; LYNCH, A. (2010): "Impact of service-oriented architecture on enterprise systems, organizational structures, and individuals", IBM Systems Journal, vol. 44, n. 4: 691-708. DOI: $10.1147 / \mathrm{sj} .444 .069$.

BORGES, F.; SILVA, P.C. (2012): “A framework for processing of business financial rules", paper presented at the 18th Brazilian Symposium on Multimedia and the Web (WebMedia 2012), São Paulo, Brazil.

BRAUER, B.; KLINE, S. (2005): "SOA governance: A key ingredient of the adaptive enterprise". http://bit.ly/18qStMJ 
CHEN, R.S.; SUN, C.M. (2007): “A collaborative continuous auditing model under service-oriented architecture environments", paper presented at the 6th WSEAS International Conference on E-ACTIVITIES, Tenerife, Spain.

CRUZ, M.S.H.; SILVA, P.C.; SILVA, L.G.C.; SANTOS, A.A. (2008): "The XBRL framework", paper presented at the 5th International Conference on Information Systems and Technology Management, São Paulo, Brazil.

ERL, T. (2009): Service-Oriented Architecture (SOA): Concepts, Technology, and Design, Ed. Prentice Hall, 9th Edition.

GLUCHOWSKI, P.; PASTWA, A. (2007): "Process and technical design of integrated solution for (semi) automated Basel II-reporting using XBRL and Web services", New Dimensions of Business Reporting and XBRL, Ed. Deutscher Universitäts-Verlag, GWV Fachverlage GmbH, Wiesbaden: 211-233.

GRI (2013): “Global Reporting Initiative XBRL Taxonomy”. http://bit.ly/1aHw1P6

HM REVENUES \& CUSTOMS - UK GOVERNMENT (2009): "Update to impact assessment of HMRC online services - Increasing use of online filing and electronic payment". http://www.hmrc.gov.uk/ria/carter-ia-final1.pdf.

HOJAJI, F.; SHIRAZI, M.R.A. (2010): "SOA governance: A new SOA governance framework based on COBIT", paper presented at the 3rd Computer Science and Information Technology (ICCSIT).

HTTP (1998): http://www.w3.org/standards/techs/http\#w3c_all.

IXBRL (2010): "What you need to know about iXBRL". http://bit.ly/18JHAvc

JOSUTTIS, N.M. (2007): SOA in Practice: The Art of Distributed System Design (Theory in Practice), Ed. O Really Media.

MARZULLO, F.P. (2009): SOA na prática inovando o seu negócio por meio de soluções orientadas a serviço. Ed. Novatec, ISBN: 9788575222010.

NIEMANN, M.; ECKERT, J.; REPP, N.; STEINMETZ, R. (2008): “Towards a generic governance model for service-oriented architectures", paper presented at the 14th Americas Conference on Information Systems. 
OTT, C.; KORTHAUS, A.; BÖHMANN, T.; ROSEMANN, M.; KRCMAR, H. (2010): "Towards a reference model for SOA governance", paper presented at the Advanced Information Systems Engineering CAiSE Forum.

QIUSHI, C.; ZUOMING, H.; JIBING, H. (2012): “A collaborative computer auditing system under SOA-based conceptual model", paper presented at the Fifth International Conference on Machine Vision (ICMV 2012): Algorithms, Pattern Recognition, and Basic Technologies.

SILVA, P.C. (2003): "Explorando linguagens de marcação para representação de relatórios financeiros", Master Degree Thesis - Salvador University. http://bit.ly/IIrvJD

SILVA, P.C.; RICCIO, E.L.; GRAMACHO, M.C. (2005): XBRL - A divulgação de informações empresariais, Ed. Ciência Moderna.

SOAP (2007): http://www.w3.org/standards/techs/soap\#w3c_all

UDDI (2004): “UDDI Version 3.0.2”. http://bit.ly/1gLCIFn

WALDMAN, D. (2009): “Cenários e etapas para implantação SOA”. http://bit.ly/IIryoy

WSDL (2007): http://www.w3.org/standards/techs/wsdl\#w3c_all

XBRL (2003): http://www.xbrl.org/SpecRecommendations

XLINK (2001): http://www.w3.org/TR/xlink

XML (2006): http://www.w3.org/standards/techs/xml\#w3c_all

XML Schema (2004): http://www.w3.org/TR/xmlschema-1

XSLT (1999): http://www.w3.org/TR/xslt

YE, H.; CHEN, S.; GAO, F. (2008): "On Application of SOA to Continuous Auditing”, WSEAS TRANSACTIONS on COMPUTERS, vol. 5, n. 7: 532-541. 